\title{
QoE-driven Unsupervised Image Categorization for Optimized Web Delivery
}

\author{
[Short Paper]
}

\author{
Parvez Ahammad, Brian Kennedy, Padmapani Ganti, Hariharan Kolam \\ Instart Logic Inc., 2307 Leghorn Street, Mountain View, CA, USA. \\ parvez@ieee.org; \{bkennedy, pganti, hkolam\}@instartlogic.com.
}

\begin{abstract}
Due to rapid growth in the richness of web applications and the multitude of wireless devices on which web content can be consumed, optimizing the content delivery of dynamic web applications represents an important technical challenge. One of the keys to solving the wireless web performance puzzle is that of efficient image content delivery. Since the critical factor in image delivery is the enduser experience, we propose a simple quantitative metric for characterizing the Quality of Experience (QoE) for any given image that gets sent through a web delivery service (WDS) pipeline. This quantitative signature, termed VoQS (variation of quality signature), allows any two arbitrary images to be compared in the context of web delivery performance. We then use VoQS in conjunction with an unsupervised learning algorithm to group similarly performing images into coherent groups for optimized web delivery. Using a large database of images compiled from multiple content providers and diverse device-optimized formats, we demonstrate that our approach allows large image databases to be efficiently parsed into coherent groups in a contentdependent and device-targeted manner for optimized image content delivery. Our approach significantly decreases the average bits per image that need to be delivered across large image databases (by $43 \%$ in our experiments) while preserving the perceptual quality across the entire image database. We also discuss how such a categorization approach can be leveraged for real-time web delivery of novel image data.
\end{abstract}

\section{Categories and Subject Descriptors}

H.3.5 [Online Information Services ]: Web-based services; I.5.3 [Pattern Recognition]: Clustering-algorithms, similarity measures; I.4.7 [Image Processing and Computer Vision]: Feature measurement

\section{Keywords}

Web delivery service (WDS); Quality of experience (QoE); Image categorization; Multimedia delivery; Unsupervised clustering

Permission to make digital or hard copies of part or all of this work for personal or classroom use is granted without fee provided that copies are not made or distributed for profit or commercial advantage, and that copies bear this notice and the full citation on the first page. Copyrights for third-party components of this work must be honored. For all other uses, contact the owner/author(s). Copyright is held by the author/owner(s).

MM'14, November 3-7, 2014, Orlando, Florida, USA.

ACM 978-1-4503-3063-3/14/11.

http://dx.doi.org/10.1145/2647868.2654982 .

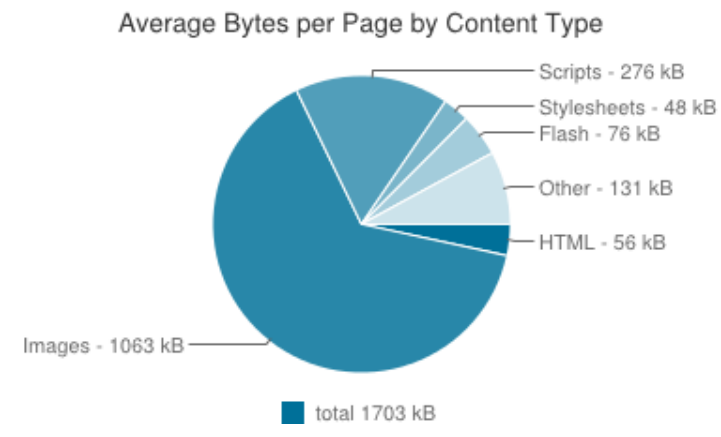

Figure 1: Content types across internet webpages: March 2014. [7].

\section{INTRODUCTION}

A key feature of present day internet is the ubiquitous presence of highly dynamic web applications and diverse set of mobile devices with sophisticated displays. The average size of the internet webpages has been steadily increasing (with a 50\% increase from May 2012 to June 2013) [14] and the average load times of image-rich websites has also been on the rise. Images represent the most significant portion of data content on an average webpage online [7] (see Figure 1). Consider a web delivery service (WDS) that serves millions of images from content providers to a large variety of internet-enabled devices. When a user attempts to access a webpage containing some images on her internet-enabled device, the WDS quickly needs to deliver the images to the user's device in a manner that maximizes the $\mathrm{QoE}$ for the user. In this context, there are two potential modes of delivery for the requested images, namely the full-download mode and the partial-download mode. In the full-download mode, the web application (such as browser) downloads the entire image content onto the user's device and then displays the image. In the context of dynamic multimedia-rich content accessed in a mobile environment, this can lead to unacceptable transfer times and low QoE for user. In contrast, in partial-download mode, the web application starts to render the image on the user device as soon as a portion of the image file is buffered [12]. Due to shorter latencies in initiating the display of image content and an efficient prioritization of bandwidth, the partial-download mode promises significant improvement in QoE.

Several critical problems arise for practical image delivery using partial-download mode on a WDS: (a) lack of a universally applicable threshold for determining the deliverable image buffer size, (b) lack of a quantitative definition for QoE for a given image, and (c) challenge of having to de- 
cide a relevant threshold for every single image in large-scale WDSs.

\subsection{Related work}

While there is a significant body of research in computer vision and image processing fields on image categorization $[8$, 3 ], very little of that work deals with the context of quality degradation of images as they pass through a web delivery pipeline. Our work draws inspiration from the image quality assessment literature $[9,15]$ and extends it to a novel problem domain. The existing industrial solutions for lowering the network footprint of image data, termed FEO (frontend optimization) methods, typically achieve lower network footprint by reducing the quality of images in the database by transcoding the images while lowering the compression quality of the images (among other optimization tricks) to adapt to the network constraints [13]. For the remainder of the paper, we will refer to the compression quality adjustment based procedures as FEO-CQ approaches. FEO-CQ approaches do not take into account the diversity of images in a database and can only be optimal when they are applied in a content-aware manner to every single image in the corpus one at a time (thus being very inefficient).

In this paper, we focus on the partial-download mode of image content delivery, and propose a simple quantitative measure for QoE of image delivery on the web, termed VoQS (variation of quality signature). Using VoQS, we then demonstrate an unsupervised image categorization solution that can coherently group large datasets of images into clusters of similar QoE, thus enabling an efficient content-aware image delivery solution across large-scale WDS.

\section{PROPOSED APPROACH}

Our proposed approach has an offline component and an online component (see Figure 2). Offline computation involves computing VoQ signatures for large database of images, using the VoQS based similarity (equation 6) to cluster the database and finding the best exemplars for each cluster. Online computation involves computing VoQS for the incoming image, comparing it to the VoQS of the stored exemplars to find the nearest neighbor and selecting an appropriate transmission buffer size based on the nearest exemplar. In the following sections, we provide further explanation of the formulations used in our pipeline.

\subsection{A QoE signature for web delivery}

Let us start by observing that the key parameter in the partial-download mode of image-delivery is the threshold $(t)$ selected for initial rendering of the image. For simplicity, we treat it as a percentage of the image size, thus $0 \leq t \leq$ 100. Let's denote variation of quality signature (VoQS) of a delivered image $I$ as $Q(I)$, and an image rendered at delivery threshold $t$ as $I_{t}$. Note that:

$$
Q(I) \triangleq\left[\bar{q}\left(I_{10}\right), . ., \bar{q}\left(I_{t}\right), . . \bar{q}\left(I_{100}\right)\right]
$$

such that $\bar{q}\left(I_{100}\right) \triangleq 1$ and $\bar{q}\left(I_{0}\right) \triangleq 0$ and $t$ is the discrete delivery cut-off point where $0 \leq t \leq 100$.

Given an original reference image $I$ and its thresholded(noisy) version $I_{t}$, one can measure $\bar{q}\left(I_{t}\right)$ in several ways. A key requirement for $\bar{q}\left(I_{t}\right)$ in a WDS framework is that it needs to be efficient to compute while capturing the perceptual similarity between $I$ and $I_{t}$.

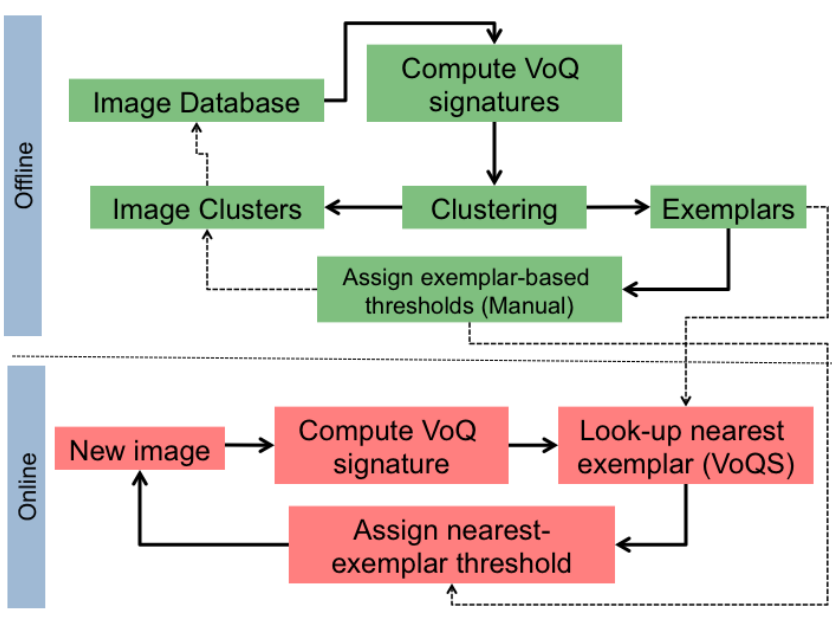

Figure 2: Flow chart of our proposed approach.

Computing the value of $\bar{q}\left(I_{t}\right)$ is closely related to the problem of image quality assessment, which has been a fundamental problem in human visual perception and computer vision literature [5]. While one reasonable way to estimate $\bar{q}\left(I_{t}\right)$ is by soliciting subjective feedback from several users [2], such an approach does not scale up to large image datasets, and can be potentially very time-consuming. Objective measurement of image quality is an active area of research, and many proposals exist without a clear winner so far. In our work, we compute $\bar{q}\left(I_{t}\right)$ through a combination of peak signal-to-noise ratio (PSNR) [9] and structural similarity metric (SSIM) [15]. While PSNR is amenable to efficient computation, SSIM tries to explicitly model the function of human visual system. Let us denote PSNR of a streamed image $I_{t}$ as $p\left(I_{t}\right)$ and SSIM of the streamed image as $s\left(I_{t}\right)$. Then,

$$
\begin{array}{r}
e\left(I, I_{t}\right)=\frac{1}{N} \sum_{i=1}^{N}\left(\left(I^{i}-I_{t}^{i}\right)^{2}\right) \\
p\left(I_{t}\right)=10 \log _{10} \frac{l^{2}}{e\left(I, I_{t}\right)} \\
s(x, y)=\left\{\frac{2 \mu_{x} \mu_{y}+C_{1}}{\mu_{x}^{2}+\mu_{y}^{2}+C_{1}} \cdot \frac{2 \sigma_{x} \sigma_{y}+C_{2}}{\sigma_{x}^{2}+\sigma_{y}^{2}+C_{2}} \cdot \frac{\sigma_{x y}+C_{3}}{\sigma_{x} \sigma_{y}+C_{3}}\right\}[15] \\
\bar{q}\left(I_{t}\right)=\left[p\left(I_{t}\right), s\left(I_{t}\right)\right]
\end{array}
$$

where $x$ and $y$ are corresponding small aggregating moving window regions chosen in $I$ and $I_{t}$ respectively (see [15] for $s\left(I_{t}\right)$ computation details). In practice, it is important to normalize the components of $Q(I)$ (such as PSNR or SSIM), so we compute z-scores [1] of each component of $Q(I)$ across the entire image database and use them for our computations.

\subsection{Offline QoE driven image categorization}

The variation of quality signature (VoQS) defines a metric space for the large-scale database of images, where the similarity between any two images $I^{\prime}$ and $I^{\prime \prime}$ is defined as:

$$
\operatorname{sim}\left(I^{\prime}, I^{\prime \prime}\right)=-\sqrt{\sum\left(Q\left(I^{\prime}\right)-Q\left(I^{\prime \prime}\right)\right)^{2}}
$$

This formulation allows a quantitative comparison between any two arbitrary images in the context of web content delivery. 


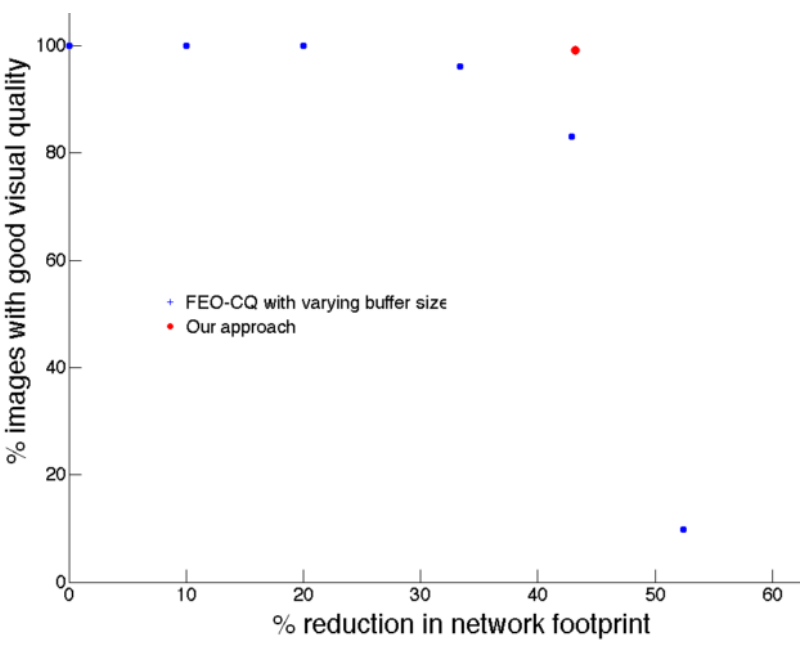

Figure 3: Results: Trade-off between overall \% reduction in network footprint vs. \% of images with good quality; Blue dots show results for FEO-CQ approach with different buffer size settings and Red dot shows our result.

We used affinity propagation algorithm [4] in conjunction with the image similarity measure defined above to find QoE-driven image clusters from the database. While other unsupervised techniques such as K-means clustering [6] or EM algorithm [11] can be used for this purpose, affinity propagation has the advantage of not requiring a pre-specification of the number of expected clusters. Affinity propagation's complexity is quadratic in the number of images, thus making it unsuitable for cases where the image database contains several millions of images. In such cases, the practical choice is to use K-means algorithm, which has linear computational complexity. The length of the VoQS vector also linearly impacts the computational complexity of the algorithm (both in the online and offline computations) - so care must be taken in choosing the number of quantized steps $t$ (see equation 1).

\subsection{Online buffer estimation for novel images}

Categorization via affinity propagation [4] readily provides an exemplar (best representative) image for each derived cluster, which can be used for manually determining the appropriate buffer size for the entire cluster. This simplifies the problem of threshold selection (to decide the buffer size) for large-scale image databases by reducing it to decisions on a few chosen exemplar images. Assuming that an offline categorization step has been computed on a large-scale WDS image database, when a novel image needs to be delivered, one can simply compute its VoQS measure and compare it to the VoQS measures of the offline computed exemplars. The buffer size for the novel image is chosen using efficient nearest-neighbor look-up among the offline computed exemplars.

\section{EXPERIMENTAL RESULTS}

\subsection{QoE driven categorization of image datasets}

To test the scalability and general applicability of our proposed method, we collected a dataset of 2238 images sampled from multiple content providers, servers and vendors

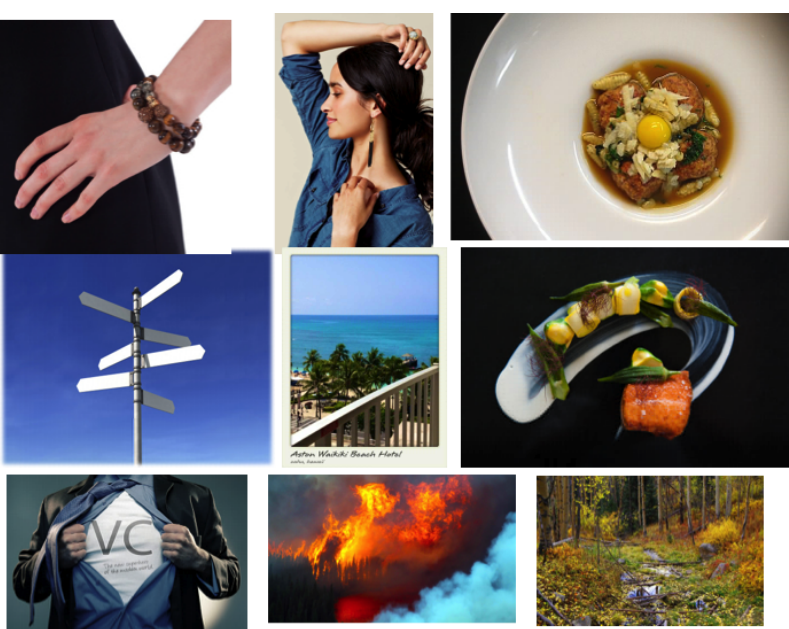

Figure 4: Results: Examples from an algorithmically discovered image cluster that tolerates buffer size of $40 \%$ of the original file size (thus enabling a significantly smaller network footprint) with minimal loss of QoE (dataset available upon request).

(see Figure 4). All the images used in our experiments are at least $10 \mathrm{~KB}$ or above. We evaluated the performance as follows:

1. offline: Compute VoQS for each image and perform unsupervised categorization of the entire dataset.

2. offline: Assign the optimal delivery threshold (buffer size) for each cluster based on the cluster exemplar alone.

3. offline: Check all the thresholded images for every cluster and estimate the images that render poorly at the selected buffer size. Error rate is estimated as the percentage of images that rendered poorly given the assigned buffer size determined via our QoE driven categorization algorithm.

Using our proposed method, we needed to only manually select buffer sizes for about 40 exemplar images in order to assign appropriate buffer sizes for the entire set of 2238 images. We found that the optimal buffer sizes ranged from $50 \%$ of the original image size to $80 \%$ of the original image size. Over the entire set of 2238 images, we found that 18 images were assigned a buffer value that rendered the thresholded version slightly noticeable in its perceptual degradation compared to the original image. This amounts to $0.8 \%$ error for the entire database while allowing a very efficient processing (approximately two orders of magnitude faster). Using the optimal buffer sizes estimated through our approach for the current experimental dataset, the network footprint can be lowered from $90,024 \mathrm{~KB}$ to $51,121 \mathrm{~KB}$ - thus resulting in $43.2 \%$ improvement in bandwidth usage.

Compared to the FEO-CQ approach of selecting one bestguess compression parameter for the entire image dataset, our approach delivered a superior trade-off between overall reduction in network footprint for the number of images delivered with good quality (see the graph in Figure 3 ). It is worth noting that our approach finds the a near-optimal solution that is well-adapted to the underlying image quality distribution in the given WDS image database.

Using our approach, it is also relatively easy to discover clusters of images that show very little perceptual degra- 


\begin{tabular}{|c||c|c||c|c|}
\hline Categories & Smartphone & Laptop & Tablet & Desktop \\
\hline \hline Smartphone & $100 \%$ & $0 \%$ & $0 \%$ & $0 \%$ \\
\hline Laptop & $0 \%$ & $100 \%$ & $0 \%$ & $0 \%$ \\
\hline Tablet & $0 \%$ & $0 \%$ & $100 \%$ & $0 \%$ \\
\hline Desktop & $0 \%$ & $0 \%$ & $0 \%$ & $100 \%$ \\
\hline
\end{tabular}

Table 1: Confusion matrix: QoE driven categorization results for device-optimized images $(\mathrm{N}=28)$

dation despite very stringent buffer size allocation. These clusters are of particular interest since they allow maximum savings in bandwidth and time while having no effect on user perception of image quality (see Figure 4 for example).

\subsection{Categorization of device-optimized images}

The ability to categorize and select images that are appropriately targeted for a particular device display format has significant commercial value in today's mobile web market. In order to evaluate the suitability of our algorithmic pipeline for this application, we collected a small dataset of images [10] that were optimized for viewing on devices with different resolutions (e.g.: smart phone, tablet device, computer monitor etc.).

In our QoE driven categorization experiments using this device-optimized image dataset $(\mathrm{N}=28)$, the proposed algorithm perfectly separated the images into the four deviceoptimized categories, namely: tablet, smartphone, desktop and laptop (see Table 1). Since our approach does not explicitly model the variations in device-types, our results suggest that it is possible to optimize device-targeted image delivery in an automated fashion using our proposed approach. In scenarios where feedback from user-device is available to the WDS, this may enable efficient retargeting of the image content based on the device-type. The proposed framework may also be useful in designing automated solutions for delivering responsive images in dynamic content delivery scenarios.

\section{SUMMARY AND FUTURE DIRECTIONS}

In this paper, we tackled the issue of reducing the network capacity required to deliver images to a variety of internetenabled devices. We presented a mechanism that allows unsupervised clustering of images into groups with similar quality variation signatures. Assuming progressive image encoding that allows rendering after partial download, our approach can determine when the image download process can be cut off without degrading image quality noticeably. As part of our approach, we proposed a quantitative quality variation measure, VoQS, which allows comparison of QoE between any two arbitrary images in the context of a web delivery service pipeline. Our algorithm would be run over an existing set of images offline to calculate clusters and then new images can be "compared" in real-time to reference images per cluster to determine efficiently to which cluster they belong. Our approach allows automated processing of large image WDS databases in a content-aware manner, and can further improve the existing web delivery optimization methods. Our approach also enables efficient estimation of optimal buffer sizes for large-scale image databases and enables significant bandwidth savings across the overall WDS network.

While VoQS is simple and intuitive, it is composed of components such as SSIM, which themselves can be further improved. In particular, components related to SSIM can be slow to compute and this can be made more efficient in future. Supervised approaches that use some groundtruth information to improve the VoQS definition as well as the similarity computations are another future avenue of research. The overall algorithmic approach proposed in this paper for quality based categorization and web delivery can be easily generalized to alternative choices of quality variation measures in future.

\section{REFERENCES}

[1] A. JaIn, K. N., AND Ross, A. Score normalization in multimodal biometric systems. Pattern recognition 38, 12 (2005), 2270-2285.

[2] BT.500-11, I.-R. R. Methodology for the subjective assessment of the quality of television images. International Telecommunication Union, Geneva, Switzerland, 2002.

[3] Chen, Y., And Wang, J. Image categorization by learning and reasoning with regions. The Journal of Machine Learning Research 5 (2004), 913-939.

[4] Frey, B., AND Dueck, D. Clustering by passing messages between data points. Science 315 (2007), 972-976.

[5] Gregory, R. Eye and brain: the psychology of seeing. Oxford University Press (June 1998).

[6] Hartigan, J. A., And Wong, M. A. Algorithm AS 136: A k-means clustering algorithm. Applied statistics (1979), 100-108.

[7] HTTPARchive. http://httparchive.org/interesting.php?a=All, 2014.

[8] J. Shotton, M. J., And Cipolla, R. Semantic texton forests for image categorization and segmentation. Proceedings of IEEE Conf. on Computer Vision and Pattern Recognition (CVPR) (2008).

[9] Marmolin, H. Subjective MSE measures. IEEE Trans. on Systems, Man, and Cybernetics 16, 3 (1986).

[10] MediaQueries. http://mediaqueri.es/about/, 2014.

[11] P. Bradley, U. F., And Reina, C. Scaling EM (expectation-maximization) clustering to large databases. Microsoft Research Technical Report MSR-TR-98-35 (1998).

[12] Rauschenbach, U., And Schumann, H. Adaptive image transmission. Proceedings of International Conf. in Central Europe on Computer Graphics and Visualization (WSCG) (1997), 434-443.

[13] S. Chandra, C. E., And Vahdat, A. Differentiated multimedia web services using quality aware transcoding. Proceedings of Nineteenth Annual Joint Conference of the IEEE Computer and Communications Societies (INFOCOM) 2 (2000), 961-969.

[14] WeBPERFormanceTODAY. http://www. webperformancetoday.com/2013/06/05/webpage-growth-2010-2013/, 2013.

[15] Z. Wang, A. Bovik, H. S., And Simoncelli, E. Image quality assessment: from error visibility to structural similarity. IEEE Trans. on Image Processing. 13, 4 (2004), 600-612. 